\title{
Brief Announcement: How Fast Reads Affect Multi-Valued Register Simulations
}

\author{
Soma Chaudhuri \\ Iowa State University \\ chaudhur@iastate.edu
}

\author{
Reginald Frank \\ Texas A\&M University \\ reginaldfrank77@tamu.edu
}

\author{
Jennifer L. Welch \\ Texas A\&M University \\ welch@cse.tamu.edu
}

\begin{abstract}
We consider the problem of simulating a $k$-valued register in a waitfree manner using binary registers as building blocks, where $k>2$. We show that for any simulation using atomic binary base registers to simulate a safe $k$-valued register in which the read algorithm takes the optimal number of steps $\left(\log _{2} k\right)$, the write algorithm must take at least $\log _{2} k$ steps in the worst case. A fortiori, the same lower bound applies when the simulated register should be regular. Previously known algorithms show that both these lower bounds are tight. We also show that in order to simulate an atomic $k$-valued register for two readers, the optimal number of steps for the read algorithm must be strictly larger than $\log _{2} k$.
\end{abstract}

\section{CCS CONCEPTS}

- Theory of Computation $\rightarrow$ Concurrency.

\section{KEYWORDS}

register simulations, wait-freedom

\section{ACM Reference Format:}

Soma Chaudhuri, Reginald Frank, and Jennifer L. Welch. 2019. Brief Announcement: How Fast Reads Affect Multi-Valued Register Simulations. In 2019 ACM Symposium on Principles of Distributed Computing (PODC '19), July 29-August 2, 2019, Toronto, ON, Canada. ACM, New York, NY, USA, 3 pages. https://doi.org/10.1145/3293611.3331580

\section{INTRODUCTION}

We consider the problem of how to use a collection of shared readwrite registers with certain properties to simulate a shared readwrite register with stronger properties in a wait-free manner. Each simulated read or write operation can access some of the building block, or "base", registers. In a wait-free simulation, each simulated operation terminates in a finite number of steps by the invoking process, regardless of the behavior of the other processes, which may be arbitrarily fast, or arbitrarily slow, or even crash. Lamport formalized the notions of safe, regular, and atomic registers, proposed a formal model, and provided numerous algorithms for simulating certain kinds of registers out of other kinds [6].

Simulating a $k$-valued register using binary base registers, where $k>2$, has been studied in, e.g., [3, 4, 6, 8], while generalizations for simulating large registers out of small registers appear in $[1,2,7]$.

Permission to make digital or hard copies of part or all of this work for personal or classroom use is granted without fee provided that copies are not made or distributed for profit or commercial advantage and that copies bear this notice and the full citation on the first page. Copyrights for third-party components of this work must be honored For all other uses, contact the owner/author(s).

PODC '19, July 29-August 2, 2019, Toronto, ON, Canada

(c) 2019 Copyright held by the owner/author(s).

ACM ISBN 978-1-4503-6217-7/19/07.

https://doi.org/10.1145/3293611.3331580
Regardless of the consistency conditions of the simulated register and the base registers, the number of steps taken by the simulated read must be at least $\log _{2} k$ [4]. However, little work has been done in exploring tradeoff results in the optimal number of base registers or the optimal number of steps taken by the simulated write when the simulated read is limited to $\log _{2} k$ steps. The only previous work we are aware of is an asymptotic result that, for the regular case, if the simulated read takes $O(\log k)$ steps, then the simulated write must take $\Omega(\log k)$ steps [5].

In this paper we consider what happens when the simulated read is limited to $\log _{2} k$ steps. In the safe and regular cases, we show that this restriction results in a lower bound of $\log _{2} k$ on the number of steps that the simulated write must take. These lower bounds are tight due to algorithms in [6] for the safe case and [4] for the regular case. In the atomic case we show that, if there are at least two readers, no such algorithm is possible, thus giving a lower bound of $1+\log _{2} k$ on the number of steps by the simulated read. This bound is not tight as the best known upper bound is $1+2 \cdot \log _{2} k[5]$.

\section{MODEL AND DEFINITIONS}

The goal is to simulate a $k$-valued register $\mathbb{X}$, with value set $V$, $|V|=k>2$, that supports $n$ readers and one writer. We assume $k$ is a power of 2 . When the $i$-th reader invokes a READ on $\mathbb{X}$, a read process, denoted $R P_{i}, 1 \leq i \leq n$, performs some computation and eventually outputs a RETURN with a value in $V$ to the reader. Similarly, when the writer invokes a WrITE with a value in $V$ on $\mathbb{X}$, the write process, denoted $W P$, performs some computation and eventually outputs an Ack to the writer. The read and write processes communicate with other through a finite set $\mathbb{B}$ of atomic base registers, each with a binary value set. Since the base registers are atomic, we model the read and write operations on them as occurring instantaneously. The values appearing in the RETURNs should satisfy one of the following consistency conditions [6]: safety (each simulated read that does not overlap a simulated write must return the value of the latest preceding simulated write), regularity (each simulated read must return the value of an overlapping simulated write or of the latest preceding simulated write), or atomicity (it should appear as if each simulated operation takes place instantaneously at some point between its invocation and response). Furthermore, as long as any particular process keeps taking steps, it should provide responses to the invocations on $\mathbb{X}$ no matter how the other processes are scheduled.

All bounds are worst-case.

\section{SAFE AND REGULAR: STEP LOWER BOUND FOR WRITE WHEN READ IS FAST}

An easy extension to a result in [4] shows: 
THeOREM 3.1. For any wait-free simulation of a safe $k$-valued register using atomic binary registers, the number of steps in the simulated read is at least $\log _{2} k$.

The main result of the section is next.

Theorem 3.2. For any wait-free simulation of a safe $k$-valued register using atomic binary registers, if the simulated read uses at most $\log _{2} k$ reads, then the simulated write uses at least $\log _{2} k$ writes.

Proof. Let $\mathbb{A}$ be any such simulation and $V$ be the value set of the simulated register, $|V|=k$.

For each $i, 1 \leq i \leq n$, we recursively define the decision tree of read process $R P_{i}$, denoted $T_{i}$, as follows. The purpose is to capture which base registers are read during the first simulated read that $R P_{i}$ executes in any execution. Each vertex in $T_{i}$ is labeled with either a base register or a value in $V$. The basis step defines the root of $T_{i}$ as a vertex labeled with the first base register read in the first simulated read performed by $R P_{i}$. For the inductive step, let $x$ be a vertex in $T_{i}$ labeled with register $r$. If the code tells $R P_{i}$ to read base register $s$ next after reading 0 (resp., 1) from $r$, then give $x$ a vertex as its left (resp., right) child labeled with $s$. If the code tells $R P_{i}$ to do $\operatorname{Return}(v)$ next after reading 0 (resp., 1$)$ from $r$, then give $x$ a vertex as its left (resp., right) child labeled with $v$. Continue until all leaves of $T_{i}$ are labeled with values.

For each $v \in V$ and each $i, 1 \leq i \leq n$, define $\sigma_{v}^{i}$ as the execution of $\mathbb{A}$ in which a simulated write of $v$ is performed by WP followed by a simulated read by $R P_{i}$. By correctness (i.e., safety) of $\mathbb{A}$, the simulated read must return $v$.

Lemma 3.3. For each $i, 1 \leq i \leq n, T_{i}$ is a complete binary tree with exactly $k$ leaves in which every leaf is at depth $\log _{2} k$ and every root-to-leaf path $\pi$ corresponds to the sequence of base registers read during the simulated read in $\sigma_{v}^{i}$, where $v$ is is the label of the leaf in $\pi$.

Proof. Fix $i, 1 \leq i \leq n$. The existence of $\sigma_{v}^{i}$ for each $v \in V$ implies that $T_{i}$ must have at least $k$ leaves. By the assumption that the step complexity of the simulated read is at most $\log _{2} k$, every root-to-leaf path in $T_{i}$ has at $\operatorname{most} \log _{2} k$ internal vertices. Since $T_{i}$ is a binary tree, basic facts from graph theory imply that $T_{i}$ has exactly $k$ leaves and each leaf is at depth exactly $\log _{2} k$. Thus the root-to-leaf path $\pi$ ending at the leaf labeled $v$ corresponds to the sequence of base registers read during the simulated read in $\sigma_{v}^{i}$.

Note that the first simulated read by each $R P_{i}$ is invisible, i.e., contains no writes to base registers, since it must have $\log _{2} k$ reads in it and we assume that the step complexity is at $\operatorname{most} \log _{2} k$.

LEMMA 3.4. Let $\pi$ be any root-to-leaf path in $T_{i}$, for any $i, 1 \leq i \leq$ $n$. Then every internal node on $\pi$ has a unique register label.

Proof. Fix $i, 1 \leq i \leq n$. Suppose in contradiction some rootto-leaf path $\pi$ has two occurrences of the register label $r$, say at distances $a$ and $b$ from the root, with $a<b$. The tree vertex representing the $b$-th read in $\pi$ has two children, since $T_{i}$ is complete by Lemma 3.3, one of which is on $\pi$. Let $\pi^{\prime}$ be a root-to-leaf path in $T_{i}$ that is the same as $\pi$ through the $b$-th read but then diverges. By Lemma 3.3, $\pi$ corresponds to execution $\sigma_{v}^{i}$ for some $v \in V$ and $\pi^{\prime}$ corresponds to execution $\sigma_{v^{\prime}}^{i}$ for some $v^{\prime} \in V$ with $v^{\prime} \neq v$.
However, it is not possible for $\sigma_{v}^{i}$ and $\sigma_{v^{\prime}}^{i}$ to read different values from $r$ at the $b$-th read since the only process that is taking steps during the simulated reads is $R P_{i}$ : even if $R P_{i}$ writes to $r$ during the simulated read, it will write the same value in $\sigma_{v}^{i}$ as in $\sigma_{v^{\prime}}^{i}$, as nothing differs in those two executions until reaching the $b$-th read.

Now we can finish the proof of the theorem. Without loss of generality, assume that the initial value of every base register is 0 . Consider the rightmost root-to-leaf path in the decision tree $T_{1}$. By Lemma 3.3, in the corresponding execution $\sigma_{v}^{1}$, the reader $R P_{1}$ reads 1 from a base register $\log _{2} k$ times during the simulated read. By Lemma 3.4, none of the base registers read during the simulated read is a repeat. Thus during the simulated write in $\sigma_{v}^{1}$, the writer $W P$ writes 1 to the each of the $\log _{2} k$ base registers that are read during the simulated read.

Since regularity is stronger than safety, we have the following corollary to the preceding theorem:

COROLlary 3.5. For any wait-free simulation of a regulark-valued register using atomic binary registers, if the simulated read uses at most $\log _{2} k$ reads, then the simulated write uses at least $\log _{2} k$ writes.

\section{ATOMIC: STEP LOWER BOUND FOR READ}

Now we consider the atomic case. We show that when there are two read processes, then it is impossible to simulate a $k$-valued register if the simulated read takes only $\log _{2} k$ steps. The key to the proof is showing that, if the simulated read takes only $\log _{2} k$ steps, then the first simulated read by each of the read processes must start by reading the same binary register first. We use the concept of decision tree and Lemmas 3.3 and 3.4 from inside the proof of Theorem 3.1.

Theorem 4.1. No wait-free simulation of an atomic $k$-valued register using atomic binary registers can have a simulated read that takes at most $\log _{2} k$ steps, assuming there are at least two readers.

Proof. Assume in contradiction there is such a simulation $\mathbb{A}$, with readers $R P_{1}$ and $R P_{2}$. We already observed that their first simulated reads are invisible (contain no writes to base registers). Let $T_{i}$ be the decision tree of the first simulated read by $R P_{i}, i=1,2$.

LEMMA 4.2. $T_{1}$ and $T_{2}$ have the same root register.

Proof. Suppose in contradiction the root $r_{1}$ of $T_{1}$ is not equal to the root $r_{2}$ of $T_{2}$. Let $z$ be a value whose root-to-leaf path in $T_{2}$ does not include $r_{1}$. Such a value must exist since $r_{1}$ is not the root of $T_{2}$. Without loss of generality, suppose $z$ appears in the right half of the leaf level of $T_{1}$. Let $a$ be a value that appears in the left half of the leaf level of $T_{1}$.

Consider an execution $e$ consisting of two simulated writes, $W_{0}(a)$ followed by $W_{1}(z)$. After $W_{0}, r_{1}$ must equal 0 , as it's possible that $R P_{1}$ 's first simulated read is immediately after $W_{0}$, in which case it must return $a$ and thus must see 0 in $r_{1}$. A similar argument shows that after $W_{1}, r_{1}$ must equal 1 . Thus $W_{1}$ must contain a write of 1 to $r_{1}$.

Consider adding two simulated reads to $e$ to make execution $e^{\prime}$ : immediately after the first write of 1 to $r_{1}$ in $W_{1}$, insert $R P_{1}$ 's first simulated read $R_{1}$, immediately followed by $R P_{2}$ 's first simulated 
read $R_{2}$. By regularity, $R_{1}$ must return either $a$ or $z$ and since $R_{1}$ observes 1 in $r_{1}$, it must return $z$. Then by atomicity, $R_{2}$ must return $z$ as well.

Consider execution $e^{\prime \prime}$ which is obtained from $e^{\prime}$ by moving $R_{1}$ to appear immediately before the first write of 1 to $r_{1}$ in $W_{1}$ and moving $R_{2}$ to appear immediately before $R_{1}$. The only difference in the registers during the execution of $R_{2}$ in $e^{\prime \prime}$ versus $e^{\prime}$ is the value of $r_{1}$. But $r_{1}$ does not lie on the root-to-leaf path in $T_{2}$ leading to $z$, and thus $R_{2}$ will behave the same in $e^{\prime \prime}$ as it does in $e^{\prime}$ and return $z$.

Consider the occurrence of $R_{1}$ in $e^{\prime \prime}$. By atomicity, $R_{1}$ must return $z$, but $R_{1}$ reads 0 in $r_{1}$ and thus cannot return $z$, a contradiction.

By Lemma 4.2, $T_{1}$ and $T_{2}$ have the same root, call it register $r$. Let $a$ and $b$ be two "sibling" values that are in the left half of the leaf level of $T_{1}$, with $a$ to the left of $b$, and $z$ be a value that is in the right half of the leaf level of $T_{1}$. Since $T_{1}$ and $T_{2}$ have the same root $r, a$ and $b$ are also in the left half of the leaf level of $T_{2}$ and $z$ is also in the right half of the leaf level of $T_{2}$. Let $x$ be the register that is the parent of $a$ and $b$ in $T_{1}$.

Consider an execution $e$ consisting of three simulated writes: $W_{0}(a)$ followed by $W_{1}(z)$, followed by $W_{2}(b)$. We make the following observations:

- After $W_{0}$ finishes, $r=x=0$. The reason is that it is possible that $R P_{1}$ does its first simulatd read between $W_{0}$ and $W_{1}$, which must return $a$ by safety and thus $T_{1}$ must have $r=$ $x=0$.

- After $W_{1}$ finishes, $r=1$. The reason is that it is possible that $R P_{1}$ does its first simulated read between $W_{1}$ and $W_{2}$, which must return $z$ by safety and thus $T_{1}$ must have $r=1$. So during $W_{1}$ there must be at least one write of 1 to $r$.

- After $W_{2}$ finishes, $r=0$ and $x=1$. The reason is that it is possible that $R P_{1}$ does its first simulated read after $W_{2}$, which must return $b$ by safety and thus $T_{1}$ must have $r=0$ and $x=1$. So during $W_{2}$ there must be at least one write of 0 to $r$ during $W_{2}$. Also, there must be at least one write of 1 to $x$ in the interval between the beginning of $W_{1}$ and the end of $W_{2}$.

The rest of the proof has two cases, depending on whether the first write of 1 to $x$ between the beginning of $W_{1}$ and the end of $W_{2}$ occurs before or after the first write of 0 to $r$ in $W_{2}$; if before, we will show that atomicity is violated; if after, that regularity is violated.

Case 1: The first write of 1 to $x$ occurs before the first write of 0 to $r$ in $W_{2}$.

Now consider another execution $e_{1}$ in which we add a simulated read $R_{1}$ by $R P_{1}$ and a simulated read $R_{2}$ by $R P_{2}$ to execution $e$. Since simulated reads are invisible, their existence does not affect the behavior of the simulated writes in $e$.

Suppose $R_{1}$ starts after $W_{0}$ ends and reads $r$ through the parent of $x$ in $T_{1}$ before $W_{1}$ begins. Then $R_{1}$ reads $x$ immediately after the first write of 1 to $x$ (which occurs in either $W_{1}$ or $W_{2}$ ), and thus sees $x=1$. So $R_{1}$ returns $b$, as indicated by its decision tree $T_{1}$.

Now suppose $R_{2}$ starts after $R_{1}$ ends and reads $r$ before $W_{2}$ 's first write of 0 to $r$, and thus sees $r=1$. By definition of atomicity, the only legal value that $R_{2}$ can return is $b$, but by the structure of its decision tree $T_{2}$ it will not return $b$, since it read 1 from $r$. Contradiction.
Case 2: The first write of 1 to $x$ occurs after the first write of 0 to $r$ in $W_{2}$.

Now consider another execution $e_{2}$ in which we add a simulated read $R_{1}$ by $R P_{1}$ to execution $e$. As in Case 1 , since simulated reads are invisible, its existence does not affect the behavior of the simulated writes in $e$.

Suppose $R_{1}$ starts and reads $r$ immediately after the first write of 0 to $r$ in $W_{2}$, seeing $r=0$, and then $R_{1}$ completes all its reads before the first write of 1 to $x$ in $W_{2}$. The structure of the decision tree $T_{1}$ implies that $R_{1}$ cannot return $z$, since $R_{1}$ read 0 in $r$, and $R_{1}$ cannot return $b$, since $R_{1}$ read 0 in $x$. But $b$ and $z$ are the only valid return values that satisfy atomicity (or even regularity for that matter). Contradiction.

The same impossibility holds with only one read process assuming that its first two simulated reads start by reading the same binary register.

\section{CONCLUSION}

We have shown how having a fast read algorithm affects the speed of the write algorithm when simulating a safe or regular $k$-valued register and that the read algorithm must be slower in the atomic case than in the safe and regular cases. Open questions include getting rid of the need for two readers to show the $1+\log _{2} k$ lower bound on the read algorithm step complexity in the atomic case. More generally, we would like to close the gap between the upper bound of $1+2 \cdot \log _{2} k$ on this measure due to [5] and the lower bound.

\section{ACKNOWLEDGMENTS}

This work was supported in part by NSF grant 1816922.

\section{REFERENCES}

[1] Zahra Aghazadeh, Wojciech M. Golab, and Philipp Woelfel. Making objects writable. In ACM Symposium on Principles of Distributed Computing, pages 385395, 2014.

[2] Alon Berger, Idit Keidar, and Alexander Spiegelman. Integrated bounds for disintegrated storage. In 32nd International Symposium on Distributed Computing, pages 11:1-11:18, 2018.

[3] Soma Chaudhuri, Martha J. Kosa, and Jennifer L. Welch. One-write algorithms for multivalued regular and atomic registers. Acta Inf., 37(3):161-192, 2000.

[4] Soma Chaudhuri and Jennifer L. Welch. Bounds on the costs of multivalued register implementations. SIAM f. Comput., 23(2):335-354, 1994.

[5] Tian Ze Chen and Yuanhao Wei. Step optimal implementations of large singlewriter registers. In 20th International Conference on Principles of Distributed Systems (OPODIS), pages 32:1-32:16, 2016.

[6] Leslie Lamport. On interprocess communication. Part II: Algorithms. Distributed Computing, 1(2):86-101, 1986.

[7] Gary L. Peterson. Concurrent reading while writing. ACM Trans. Program. Lang. Syst., 5(1):46-55, 1983.

[8] K. Vidyasankar. Converting Lamport's regular register to atomic register. Inf. Process. Lett., 28(6):287-290, 1988. 